\title{
Dialisi Peritoneale, why not!!!
}

\section{Viviana Finato}

Dirigente medico 1o livello UOC Nefrologia e Dialisi San Miniato, Usl 11, Empoli (FI)

\author{
Peritoneal dialysis: Why not? \\ Abstract: Personal experience of a nephrologist devoted to, but not responsable of, peritoneal dialysis.
}

Key words: Peritoneal dialysis, audit

Conflict of interest: None.

Financial support: None.

Accettato: 21 Agosto 2013

Mi dedico alla Dialisi Peritoneale dal 2007, quando la mia collega è andata in pensione e mi ha lasciato questa "pesante" eredità o, almeno, così ho pensato all'inizio, perché mi potevo dedicare a questi assistiti solo in piccoli ritagli di tempo, quando i miei colleghi me lo permettevano per disponibilità di orario o, comunque, spesso fuori orario di servizio.

Percependo davvero la DP come dialisi marginale, ho pensato che tutto fosse dovuto alla mia poca conoscenza in materia, visto che, nel mio Corso di Laurea e di Specializzazione, era un argomento non trattato (e, in molte Università, continua a esserlo).

Ho deciso, pertanto, di partecipare a tutti i Congressi dove si parlasse di Dialisi Peritoneale e, soprattutto, ho iniziato a frequentare le riunioni del Gruppo di Studio di Dialisi Peritoneale e mi sono resa conto del fatto che la "comunità" peritonealista era stufa di essere il medico di serie B per il paziente di serie B.

Finalmente ho percepito la volontà di dimostrare che la Dialisi Peritoneale non deve rimanere marginale!!!

Nel mio piccolo, ho iniziato a lavorare a fianco del collega chirurgo per fargli capire che il catetere peritoneale non è un drenaggio addominale, ma è un accesso di dialisi.

Un buon accesso è la chiave del successo e, con questo slogan, i pazienti hanno iniziato ad avvicinarsi più volentieri a una metodica che spesso veniva associata a tanti lassativi per cateteri spesso dislocati.

Quindi, ho iniziato a fare il PET 2.27, poi il 3.86 e, ora, UNIPET, la dialisi incrementale, la pressione endoperitoneale e così via, e ora, da 20 pazienti, ho 40 pazienti che svolgono la DP con metodiche personalizzate ed efficienti.

Ora sono impegnata a seguire i pazienti "fragili", nell'intento di domiciliarizzare la dialisi e di renderla accessibile a coloro che, per paura di eseguire la dialisi a casa senza la presenza di personale sanitario, la rifiutano.

Sono riuscita a unire i medici dedicati alla DP della Regione Toscana, formando il gruppo Toscana DP, che ha trovato l'entusiasmo di lavorare insieme, eseguendo tre audit clinici all'anno su argomenti tratti dalle buone pratiche del Gruppo di Studio.

Per il momento, credo che la DP sia ancora una dialisi marginale, ma che possa decollare; questo solo se le Regioni, le Amministrazioni, i medici di medicina generale e i nefrologi di serie A la conosceranno meglio!!!

La possibilità di iniziare un dialogo tra colleghi peritonealisti tramite una rivista mi sembra un ottimo trampolino di lancio per aiutare quelli come me che hanno iniziato da poco e con molte difficoltà. Certamente, l'intervento dei Prof. Di Paolo e Buoncristiani mette nella condizione di pensare che, per fare DP, non basta solo l'entusiasmo, ma serve ancora tanta ricerca e, forse, solo tramite scambi culturali e l'unione di esperienze e di dati potremo veramente avere una svolta per rendere la DP non marginale.

\section{Riassunto}

Esperienza personale di un medico (non Direttore di UOC) che svolge il proprio servizio come medico dedicato ma non responsabile della DP.

Parole chiave: Dialisi Peritoneale, Audit

Dichiarazione di conflitto di interessi: L'Autore dichiara di non avere conflitto di interessi.

Contributi economici degli autori: L'Autore dichiara di non aver ricevuto sponsorizzazioni economiche per la preparazione dell'articolo.

Indirizzo degli Autori:

Dr.ssa Viviana Finato

Dirigente Medico U.O.C. Nefrologia e Dialisi

Ospedale San Miniato Alto

Piazza XX settembre

USL 11 Empoli

50053 Empoli (FI)

vivifinato@gmail.com 